\title{
UNIQUENESS OF HOMOGENEOUS DIFFERENTIAL POLYNOMIALS OF MEROMORPHIC FUNCTIONS CONCERNING WEAKLY WEIGHTED SHARING
}

\author{
DILIP CHANDRA PRAMANIK AND JAYANTA ROY
}

\begin{abstract}
In 2006 S. Lin and W. Lin [3] first defined the concept of weakly-weighted sharing of functions and proved some results on uniqueness of a meromorphic function $f$ and its $n$-th derivative $f^{(n)}$. Using this notion of weakly-weighted sharing of functions, in this paper we prove uniqueness of homogeneous differential polynomials $P[f]$ and $P[g]$ generated by meromorphic functions $f$ and $g$ respectively.
\end{abstract}

Mathematics subject classification (2010): 30D30, 30D35.

Keywords and phrases: Meromorphic functions, Nevanlinna theory, weakly-weighted sharing, differential polynomial, uniqueness.

\section{REFERENCES}

[1] W. K. Hayman, Meromorphic function, Clarendon Press, Oxford, 1964.

[2] J. D. HinchlifFe, On a result of chuang related to Hayman's alternative, Comput. Mathods Funct. Theory, 2, (2002), 293-297.

[3] S. LIN AND W. LIN, Uniqueness of meromorphic functions concerning weakly weighted sharing, Kodai. Math. J., 29, (2006), 269-280.

[4] J. T. Li AND P. Li, Uniqueness of entire functions concerning differential polynomials, Commun. Korean Math. Soc., 30, 2 (2015), 93-101.

[5] I. LAHIRI AND B. PAL, Uniqueness of meromorphic functions with their homogeneous and linear differential polynomials sharing a small function, Bull. Korean Math. Soc., 54, (2017), 825-838.

[6] D. C. PRAMANIK, Weighted set sharing and uniqueness of meromorphic functions, Konuralp Journal of Mathematics, 7, 2 (2019), 243-251.

[7] D. C. PRAMANIK AND J. ROY, Weakly weighted-sharing and uniqueness of homogeneous differential polynomials, Mat. Stud., 51, 1 (2019), 41-49.

[8] D. C. PRAMANiK AND J. RoY, Uniqueness of homogeneous differential polynomials concerning weakly weighted-sharing, Commun. Korean Math. Soc., 34, 2 (2019), 439-449.

[9] C. C. YANG, On deficiencies of differential polynomials II, Math. Z., 125, (1972), 107-112.

[10] L. YANG, Value distributions theory, Springer-Verlag, Berlin, 1993.

[11] H. X. YI AND C. C. YANG, Uniqueness theory of meromorphic functions(in Chinese), Science Press, Beijing, 1995.

[12] H. X. YI, Uniqueness theorems for meromorphic functions whose nth derivatives share the same 1-points, Complex Var. Theory Appl., 34, 4 (1997), 421-436.

[13] H. Y. XU AND Y. HU, Uniqueness of meromorphic function and its Differential polynomial concerning weakly weighted sharing, General Mathematics, 19, (2011), 101-111. 